\title{
Retraction Note to: Nuclear Protein C23 on the Cell Surface Plays an Important Role in Activation of CXCR4 Signaling in Glioblastoma
}

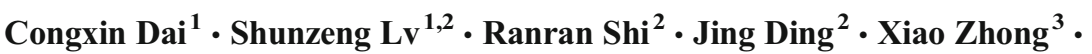 \\ Huishu Song ${ }^{2}$ - Xiaochen $\mathrm{Ma}^{2}$ - Jianzhen Fan ${ }^{2} \cdot$ Bowen Sun ${ }^{1} \cdot$ Renzhi Wang ${ }^{1}$. \\ Wenbin $\mathrm{Ma}^{1}$
}

Published online: 23 March 2017

(C) Springer Science+Business Media New York 2017

Retraction Note to: Mol Neurobiol (2015) 52:1521-1526

DOI 10.1007/s12035-014-8955-7

This article has been retracted at the request of the Editor-inChief and the Publisher per the Committee on Publication Ethics guidelines. The article shows evidence of irregularities in authorship.

During the submission process, there is strong reason to believe that the peer review process was compromised and the authors have plagiarized parts from the following article:

Shunzeng Lv, Congxin Dai, Yuting Liu, Bowen Sun, Ranran Shi, Mingzhi Han, Ruixiang Bian, Renzhi Wang, Cell Surface Protein C23 Affects EGF-EGFR Induced Activation of ERK and PI3K-AKT Pathways, Journal of Molecular Neuroscience, February 2015, Volume 55, Issue 2, pp. 519524, DOI: 10.1007/s12031-014-0375-7; Received: 26 June 2014.

As such, the validity of the content of this article cannot be verified.

The online version of the original article can be found at http://dx.doi.org/ $10.1007 / \mathrm{s} 12035-014-8955-7$

Wenbin Ma

mwbpumch@163.com

1 Department of Neurosurgery, Peking Union Medical College Hospital, Chinese Academy of Medical Sciences and Peking Union Medical College, Beijing 100730, China

2 Shandong University School of Medicine, Jinan, Shandong, China

3 Department of Obstetrics and Gynecology, Peking Union Medical Southern Medical University, Zhongshan, Guangdong, China 